\title{
Efficient and accurate approach for powder compaction problems
}

\author{
A. Pérez-Foguet, A. Rodríguez-Ferran, A. Huerta
}

Abstract In this paper, a new approach for powder cold compaction simulations is presented. A density-dependent plastic model within the framework of finite strain multiplicative hyperelastoplasticity is used to describe the highly nonlinear material behaviour; the Coulomb dry friction model is used to capture friction effects at die-powder contact; and an Arbitrary Lagrangian-Eulerian (ALE) formulation is used to avoid the (usual) excessive distortion of Lagrangian meshes caused by large mass fluxes. Several representative examples, involving structured and unstructured meshes are simulated. The results obtained agree with the experimental data and other numerical results reported in the literature. It is shown that, contrary to other Lagrangian and adaptive $h$-remeshing approaches recently reported for this type of problems, the present approach verifies the mass conservation principle with very low relative errors (less than $1 \%$ in all ALE examples and exactly in the pure Lagrangian examples). Moreover, thanks to the use of an ALE formulation and in contrast with other simulations, the presented density distributions do not present spurious oscillations.

Keywords Powder compaction, finite strain multiplicative plasticity, consistent tangent moduli, Arbitrary Lagrangian-Eulerian (ALE) formulation, density-dependent models, numerical differentiation

\section{1}

\section{Introduction}

Cold compaction processes are a key ingredient in powder forming processes. They consist in the vertical compaction through the movement of a set of punches of a fine powder material at room temperature. The process transforms the loose powder into a compacted sample with a volume reduction (and therefore a density increase) of about 2-2.5

Received: 20 March 2002 / Accepted: 15 October 2002

\author{
A. Pérez-Foguet $(\varangle)$, A. Rodríguez-Ferran, A. Huerta \\ Depart. de Matemàtica Aplicada III, \\ E.T.S. de Ingenieros de Caminos, \\ Canales y Puertos, Universitat Politècnica de Catalunya, \\ Jordi Girona 1, E-08034 Barcelona, Spain \\ e-mail: agusti.perez@upc.es
}

The partial financial support of the Ministerio de Ciencia y Tecnología (grant number DPI 2001-2204) is gratefully acknowledged. times. The design of these processes includes the definition of the initial dimensions of the sample and the movements of the punches that lead to compacted samples with uniform density distributions. In this context, efficient and reliable numerical simulations can play an important role as a complement of experimental tests.

Two ingredients are crucial for the numerical modelling of powder compaction processes: the constitutive relationship and the kinematic formulation of the problem. Several constitutive models have been proposed, including microscopic models, flow formulations and solid mechanics models, such as elastic, plastic or viscoplastic models; see Oliver et al. [1] and Lewis and Khoei [2] for a general overview and references for each type of model. One of the most common approaches is the use of elastoplastic models based on porous or frictional materials. Here, plastic models expressed in terms of the relative density and the Kirchhoff stresses are considered. The constitutive relationship is formulated within the framework of isotropic finite strain multiplicative hyperelastoplasticity $[3,4]$. This type of models has already been applied to powder compaction problems with some simplifications derived from the assumption of small elastic strains [1]. In this work, large elastic strains are included in the formulation [5]. It is shown that this does not represent any drawback from a modelling point of view. On the contrary, it allows to apply numerical techniques and material models developed for the general kinematic framework in a straightforward manner.

Up to date, a common feature of powder compaction simulations with solid mechanics constitutive models is the use of a Lagrangian kinematic formulation. This approach has shown to be adequate for problems that do not exhibit large mass fluxes among different parts of the sample (i.e. homogeneous tests). But in practical problems, as those which appear in realistic design processes, the Lagrangian approach leads to too distorted meshes $[2,6]$ or violations of the boundary limits [1]. In order to solve these problems, different $h$-adaptive procedures have been presented recently $[6,7]$. However, $h$-refinement is computationally expensive and information must be interpolated from the old mesh to the new mesh. For these reasons an Arbitrary Lagrangian-Eulerian (ALE) approach is preferred in this work. ALE formulations were first proposed for fluid problems with moving boundaries $[8,9]$. Nowadays, ALE formulations for fluid problems are widely used in forming processes. On the other hand, the ALE formulation has been successfully employed in nonlinear solid mechanics [10-14]. The ALE formulation for 
multiplicative hyperelastoplasticity recently presented by Rodríguez-Farran et al. [14] is used here.

Due to the high nonlinearity of powder compaction simulations, a common approach is the explicit treatment of both the integration of the constitutive equations and the solution of the equilibrium equation $[2,6,15]$, although, implicit schemes have also been successfully applied [1,7]. In fact, the development of robust, accurate and efficient schemes, both implicit and explicit, is still an active research topic, see for instance [16-19] among many others. Here a mixed approach is followed. The integration of the ALE constitutive equations is done by means of a fractional-step method [3]: the treatment of the Lagrangian phase is implicit and the scheme for the convective phase is explicit. The equilibrium equation is solved with an incremental-iterative approach, where only the Lagrangian phase is performed within the iterations of each load increment (the perturbation due to the integration of the convective phase is taken into account in the subsequent load increment). A key point for the efficient implicit solution of the equilibrium equation is the consistent linearization of the algorithm, which is given by the proper consistent tangent moduli [20]. The recently presented expression for density-dependent models by Pérez-Foguet et al. [5] is used here. Specific tools for two of the main issues of implicit approaches for complex elastoplastic inoc within this work: the converge at Gauss-point level is guar convergent Newton-like sche differentiation schemes ar $[18,23]$.

An outline of the paper follows. The main features of

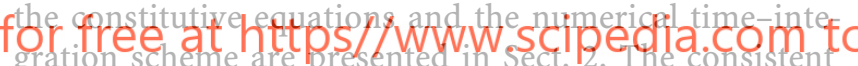
tangent moduli is presented in Sect. 3. After that, in Sect. 4, the proposed approach is applied to several representative powder compaction problems. The present results are compared with experimental data and other results of numerical simulations presented in the literature. Section 5 contains some concluding remarks.

2

\section{Problem statement}

In this section, the basis of the proposed approach is briefly presented. First, the kinematics of the ALE formulation are reviewed. After that, the finite strain elliptic elastoplastic model is described, focusing in the yield function dependence on the density. Finally, the numerical time-integration algorithm is presented.

\section{1}

\section{Kinematics}

Let $R_{X} \subset \mathbb{R}^{n_{\mathrm{dim}}}\left(n_{\mathrm{dim}}=2,3\right)$ be the material configuration of a continuum body with particles labelled by their initial position vector $\mathbf{X} \in R_{X}$. In a Lagrangian setting, $\mathbf{X}$ are used as the independent variables in the description of motion. The motion of the body is described by the one-parameter family of mappings $\boldsymbol{\varphi}_{t}: R_{X} \mapsto \mathbb{R}^{n_{\mathrm{dim}}}$ with $t \in[0, T]$. $R_{x}=\varphi_{t}\left(R_{X}\right)$ is the spatial configuration of the body at time $t$, and $\mathbf{x}=\boldsymbol{\varphi}_{t}(\mathbf{X})=\boldsymbol{\varphi}(\mathbf{X}, t) \in R_{x}$ is the current position of the material particle $\mathbf{X}$.

The key ingredient of the ALE formulation is the referential configuration $R_{\chi}$, with grid (or reference) points $\chi$ used as independent variables to describe body motion. This referential configuration $R_{\chi}$ is mapped into the material and spatial configurations by $\boldsymbol{\Psi}$ and $\boldsymbol{\Phi}$ respectively, see figure 1 . The initial position of a material particle is expressed on the reference domain as $\mathbf{X}=\boldsymbol{\Psi}(\chi, t)$, and the current position as $\mathbf{x}=\boldsymbol{\Phi}(\chi, t)$. The three mappings $\boldsymbol{\varphi}, \boldsymbol{\Phi}$ and $\boldsymbol{\Psi}$ are related by $\boldsymbol{\varphi}=\boldsymbol{\Phi} \circ \boldsymbol{\Psi}^{-1}$.

In an ALE setting, different displacement fields can be 221 defined. Two of them have special interest, the particle displacement $\mathbf{u}$ and the mesh (spatial) displacement $\mathbf{u}_{\Phi}$,

$\mathbf{u}(\mathbf{X}, t)=\mathbf{x}(\mathbf{X}, t)-\mathbf{X} \quad$ and $\quad \mathbf{u}_{\Phi}(\chi, t)=\mathbf{x}(\chi, t)-\chi$.

The particle velocity and the mesh velocity are respectively

$\mathbf{v}=\left.\frac{\partial \mathbf{x}}{\partial t}\right|_{\mathbf{x}} \quad$ and $\quad \mathbf{v}_{\text {mesh }}=\left.\frac{\partial \mathbf{x}}{\partial t}\right|_{\chi}$

where, following standard notation, $\left.\right|_{*}$ means "holding * fixed". The link between material and mesh motion is provided by the convective velocity, $\mathbf{c}=\mathbf{v}-\mathbf{v}_{\text {mesh }}$.

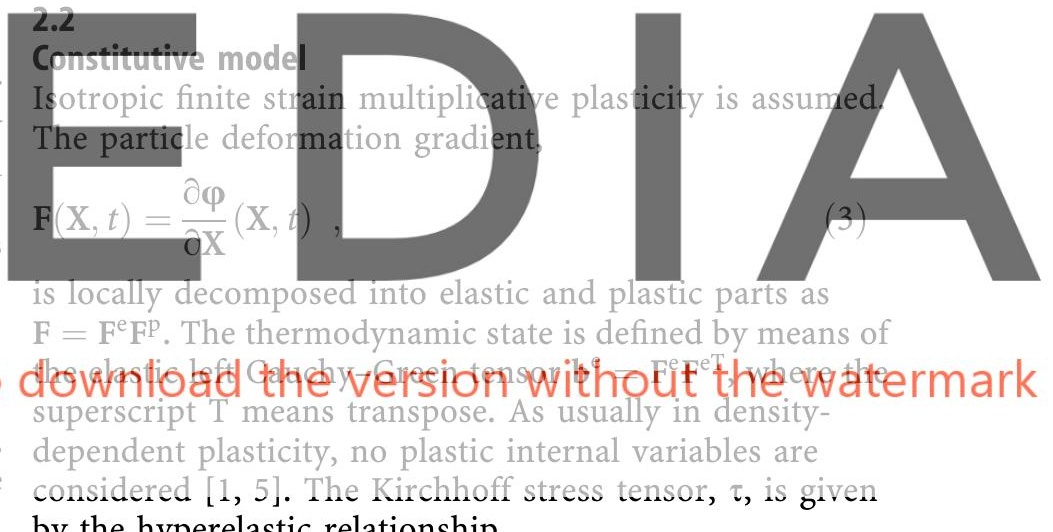
by the hyperelastic relationship

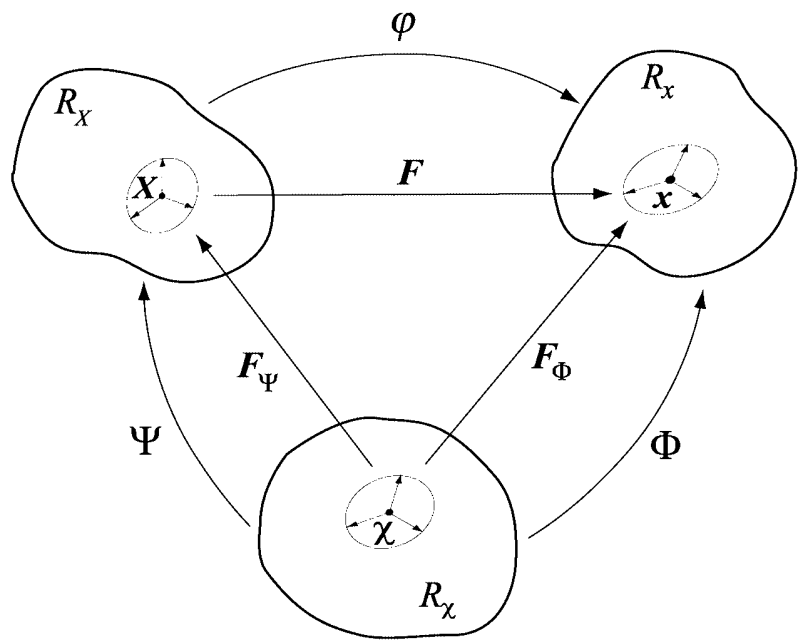

Fig. 1. Domains, mappings and deformation gradients in the ALE description 
$\tau=2 \frac{\mathrm{d} W^{\mathrm{e}}}{\mathrm{db}^{\mathrm{e}}} \mathbf{b}^{\mathrm{e}}$

where $W^{\mathrm{e}}$ is the free-energy function per unit of undeformed volume. The Cauchy stress tensor is given by

$\boldsymbol{\sigma}=\frac{\tau}{\operatorname{det}(\mathbf{F})}$.

The plastic response of the material is assumed isotropic and density-dependent. The dependence on the density is incorporated in the yield function $f(\tau, \eta)$ and the flow direction $\mathbf{m}(\tau, \eta)$ through the relative density $\eta(\mathbf{X}, t)$ of the particle $\mathbf{X}$ at time $t$. The relative density is equal to the real density of the material divided by a reference value, which usually is the solid density of the compacted material. Associative plasticity is characterized by $\mathrm{m}(\tau, \eta)=\nabla_{\tau} f(\tau, \eta)$, with $\nabla_{*}$ equal to the gradient operator with respect to the variables $*$.

The evolution of the elastic left Cauchy-Green tensor (i.e. flow rule) is defined in the ALE setting as [14]

\section{$\partial \mathbf{b}^{\mathrm{e}}$}

$\left.\frac{\partial \mathbf{b}^{\mathrm{e}}}{\partial t}\right|_{\chi}+\mathbf{c} \nabla_{\mathrm{x}} \mathbf{b}^{\mathrm{e}}-\mathbf{l b}^{\mathrm{e}}-\mathbf{b}^{\mathrm{e}} \mathbf{1}^{\mathrm{T}}=-2 \dot{\gamma} \mathrm{m}(\tau, \eta) \mathbf{b}^{\mathrm{e}}$

where $\mathbf{l}=\nabla_{\mathbf{X}} \mathbf{V}$ is the velocity gradient tensor and $\dot{\gamma}$ is the plastic multiplier. Equation (6) is complemented with the Kuhn-Tucker conditions

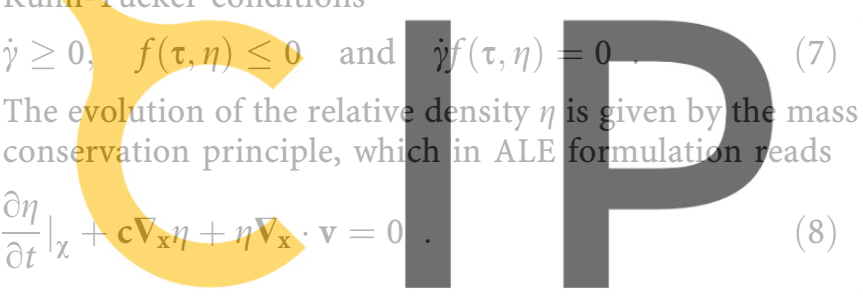

2.3

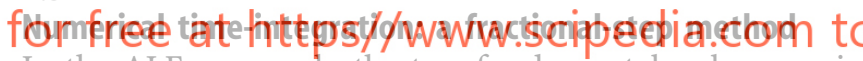
In the ALE approach, the two fundamental unknowns in every time-step $\left[{ }^{n} t,{ }^{n+1} t\right]$, with ${ }^{n+1} t={ }^{n} t+\Delta t$, are the increment of mesh displacements,

${ }^{n+1} \Delta \mathbf{u}_{\Phi}(\boldsymbol{\chi})={ }^{n+1} \mathbf{x}(\boldsymbol{\chi})-{ }^{n} \mathbf{x}(\boldsymbol{\chi})$

and the increment of particle displacements,

${ }^{n+1} \Delta \mathbf{u}\left({ }^{n} \mathbf{X}(\boldsymbol{\chi})\right)={ }^{n+1} \mathbf{X}\left({ }^{n} \mathbf{X}(\boldsymbol{\chi})\right)-{ }^{n} \mathbf{X}\left({ }^{n} \mathbf{X}(\boldsymbol{\chi})\right)$

which is referred to the particles ${ }^{n} \mathbf{X}$ associated to grid points $\chi$ at the beginning of the time-step, ${ }^{n} \mathbf{X}=\mathbf{X}\left(\chi,{ }^{n} t\right)$. The mesh displacements are obtained from an ALE remeshing algorithm. The particle displacements are found via the incremental solution of the equilibrium equation. The difference between the increments of particle and mesh displacements is the so-called increment of convective displacements,

${ }^{n+1} \Delta \mathbf{u}_{\text {conv }}(\chi)={ }^{n+1} \Delta \mathbf{u}(\chi)-{ }^{n+1} \Delta \mathbf{u}_{\Phi}(\chi)$,

which represents the relative motion between particles and grid points during the time-step.

The quantities to integrate are only $\mathbf{b}^{\mathrm{e}}$ and $\eta$, see remark 1 below. The numerical time-integration is done by means of a fractional-step method over Equations (6) and (8). Every time-step is divided into two phases: the Lagrangian phase and the convection phase.
During the Lagrangian phase convection is neglected, $\mathbf{c}=\mathbf{0}$. In that situation, Eq. (6) simplifies to the standard spatial expression of the flow rule

$\mathcal{L}_{\mathrm{v}} \mathbf{b}^{\mathrm{e}}=-2 \dot{\gamma} \mathbf{m}(\tau, \eta) \mathbf{b}^{\mathrm{e}}$,

where $\mathcal{L}_{\mathrm{v}} \mathbf{b}^{\mathrm{e}}=\left.\frac{\partial \mathbf{b}^{\mathrm{e}}}{\partial t}\right|_{\mathbf{x}}-\mathbf{l} \mathbf{b}^{\mathrm{e}}-\mathbf{b}^{\mathrm{e}} \mathbf{1}^{\mathrm{T}}$ is the Lie derivative with respect to the particle velocity; and Eq. (8) simplifies to

$\eta(\mathbf{X}, t)=\frac{\eta_{0}(\mathbf{X})}{\operatorname{det}(\mathbf{F})}$

the material expression of the Lagrangian relative density evolution. The initial conditions for equations (12) and (13) are the state at time ${ }^{n} t\left({ }^{n} \mathrm{x},{ }^{n} \mathbf{b}^{\mathrm{e}}\right.$ and $\left.{ }^{n} \eta\right)$. The problem is strain-driven, thus, the Lagrangian values ${ }^{L} \mathbf{b}^{\mathrm{e}}$ and ${ }^{L} \eta$ are obtained from ${ }^{n+1} \Delta \mathbf{u}$. Recall that ${ }^{n+1} \Delta \mathbf{u}$ is not required to be in equilibrium.

After the Lagrangian phase, an ALE remeshing algorithm is employed to compute the increment of mesh displacements ${ }^{n+1} \Delta \mathbf{u}_{\Phi}$. The algorithm is defined such as it reduces the element distortion of a pure Lagrangian approach.

During the convection phase, the convective term of the evolution equations is taken into account. The convective velocity is assumed constant in the time-step and equal to $\mathrm{c}={ }^{n+1} \Delta \mathrm{u}_{\text {conv }} / \Delta t$. The final (convected) values

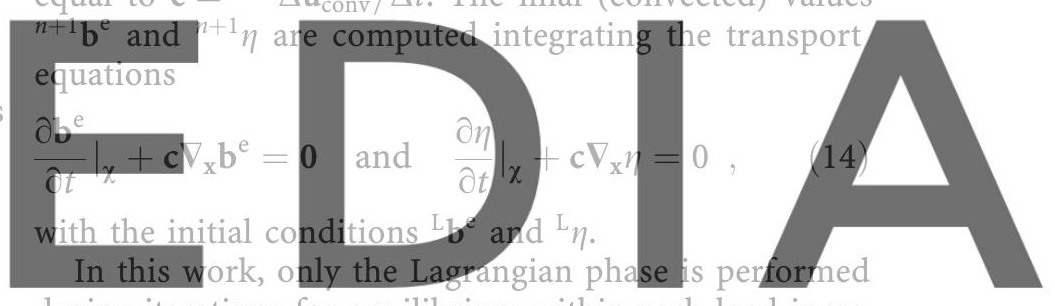
during iterations for equilibrium within each load incre ment. The remeshing and the convection are computed

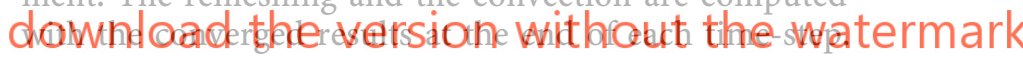
This results into a reduced computational cost overhead with respect to the pure Lagrangian approach. The equilibrium perturbation due to the remeshing and the convective time-integration of the converged results is taken into account in the subsequent load increment. Therefore, there are no accumulative errors in the results. On the other hand, as the iterative scheme is pure Lagrangian, the consistent operator is not influenced by the remeshing technique nor by the numerical time-integration scheme used in the convective phase. The overall scheme is summarized in table 1 . The main characteristics of the numerical time-integration of each phase are presented in the following two subsections.

Remark 1 In a general setting, all quantities related with the particles should be convected. This includes the material parameters and the value of $\operatorname{det}(\mathbf{F})$, in addition to $\mathbf{b}^{\mathrm{e}}$ and $\eta$ (and the plastic internal variables if they exist).

However, in the usual case that the material surfaces are tracked by the ALE remeshing algorithm and homogeneous materials are considered (as is the case in all the examples presented in this work), only the integration of $\mathbf{b}^{\mathrm{e}}$ and $\eta$ is necessary. The value of $\operatorname{det}(\mathbf{F})$ can be computed from the material expression of the relative density evolution, Eq. (13). 
Table 1. The overall ALE scheme

For every time-step $\left[{ }^{n} t,{ }^{n+1} t\right]$ :

Material phase

- Neglect convective terms

- Advance the solution iteratively in an updated Lagrangian fashion: compute the increment of particle displacements ${ }^{n+1} \Delta \mathbf{u}$ and quantities ${ }^{\mathrm{L}} \mathbf{b}^{\mathrm{e}}$ and ${ }^{\mathrm{L}} \eta$ (superscript $\mathrm{L}$ denotes Lagrangian)

\section{Remeshing}

- Compute the increment of mesh displacements ${ }^{n+1} \Delta \mathbf{u}_{\Phi}$ and the increment of convective displacements ${ }^{n+1} \Delta \mathbf{u}_{\text {conv }}$ by means of a remeshing algorithm that reduces element distortion.

- Compute the convective velocity $\mathrm{c}={ }^{n+1} \Delta \mathbf{u}_{\text {conv }} / \Delta t$

\section{Convection phase}

- Account for convective terms

- Use the Godunov-type technique to convect quantities ${ }^{\mathrm{L}} \mathbf{b}^{\mathrm{e}}$ and

${ }^{L} \eta$ into ${ }^{n+1} \mathbf{b}^{\mathrm{e}}$ and ${ }^{n+1} \boldsymbol{\eta}$

- Compute stresses ${ }^{n+1} \tau$ and ${ }^{n+1} \sigma$

\subsection{1}

Lagrangian phase

The results of the Lagrangian phase, ${ }^{\mathrm{L}} \mathbf{b}^{\mathrm{e}}$ and ${ }^{\mathrm{L}} \eta$, are computed from ${ }^{n} \mathbf{b}^{\mathrm{e}},{ }^{n} \eta$ and the incremental particle deformation gradient,

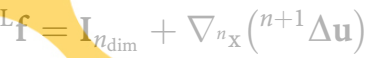

which relates the particle defor

${ }^{n} \mathrm{~F}$, and at the end of the Lagr relationship ${ }^{{ }_{\mathrm{f}}}={ }^{\mathrm{L}} \mathrm{F}\left({ }^{n} \mathrm{~F}\right.$

of order $n_{\text {dim }}$.

The relative density, $\eta$, is i

that no numerical time-integ

cause of the material expres

principle, Eq. (13), which leads to $\operatorname{det}\left({ }^{\mathrm{L}} \mathrm{F}\right)$ $\operatorname{det}\left(\mathrm{L}_{\mathrm{f}}\right.$

The value of ${ }^{\mathrm{L}} \mathrm{b}{ }^{\mathrm{e}}$ is obtained by means of the standard elastic predictor-plastic corrector split strategy applied to Eqs. (7) and (12). Remarkably, the dependence of the constitutive equations on the density does not modify the algorithm. The value of ${ }^{\mathrm{L}} \eta$ is given by Eq. (16), and therefore it plays the role of a fixed parameter.

The result of the elastic predictor step is the so-called trial state. It is defined by

${ }^{\operatorname{tr}} \mathbf{b}^{\mathrm{e}}={ }^{\mathrm{L}} \mathbf{f}^{n} \mathbf{b}^{\mathrm{eL}} \mathbf{f}^{\mathrm{T}}$.

If the trial state is admissible, $f\left(\tau\left({ }^{\mathrm{tr}} \mathbf{b}^{\mathrm{e}}\right),{ }^{\mathrm{L}} \eta\right) \leq 0$, the value of ${ }^{\mathrm{L}} \mathbf{b}^{\mathrm{e}}$ is set equal to the trial state. If it is not, a plastic corrector step is computed. Recall that the trial state, Eq. (17), does not depend on ${ }^{\mathrm{L}} \eta$, a key point in the linearization of the algorithm presented in Sect. 3.

The plastic correction step requires the approximation of the flow rule, Eq. (12). A standard approximation consists in the use of the exponential map and the backward Euler integration scheme [3]. Under the previous isotropy assumptions, this approach leads to a nonlinear system of equations with the same structure as that of infinitesimal elastoplasticity. In order to obtain this nonlinear system of equations, three vectors of $\mathbb{R}^{n_{\mathrm{dim}}}$ are defined: ${ }^{\operatorname{tr}} \boldsymbol{\varepsilon}^{\mathrm{e}},{ }^{\mathrm{L}} \boldsymbol{\varepsilon}^{\mathrm{e}}$ and ${ }^{\mathrm{L}} \bar{\tau}$. The components of ${ }^{\text {tr }} \boldsymbol{\varepsilon}^{\mathrm{e}}$ and ${ }^{\mathrm{L}} \boldsymbol{\varepsilon}^{\mathrm{e}}$ are functions of the eigenvalues of the tensors ${ }^{\operatorname{tr}} \mathbf{b}^{\mathrm{e}}$ and ${ }^{\mathrm{L}} \mathbf{b}^{\mathrm{e}}$, respectively, and the components of ${ }^{\mathrm{L}} \bar{\tau}$ are the eigenvalues of ${ }^{\mathrm{L}} \tau$ :

$$
{ }^{\star} \mathbf{b}^{\mathrm{e}}=\sum_{i=1}^{n_{\mathrm{dim}}}\left(\exp \left(\left[{ }^{*} \boldsymbol{\varepsilon}^{\mathrm{e}}\right]_{i}\right)\right)^{2} \mathbf{n}_{\mathrm{tr}}^{i} \otimes \mathbf{n}_{\mathrm{tr}}^{i} \text { and }{ }^{\mathrm{L}} \boldsymbol{\tau}=\sum_{i=1}^{n_{\mathrm{dim}}}\left[{ }^{\mathrm{L}} \overline{\boldsymbol{\tau}}\right]_{i} \mathbf{n}_{\mathrm{tr}}^{i} \otimes \mathbf{n}_{\mathrm{tr}}^{\mathrm{i}},
$$

where the superscript $\star$ refers to tr and $\mathrm{L},\left\{\mathbf{n}_{\mathrm{tr}}^{i}\right\}_{i=1, \ldots, n_{\mathrm{dim}}}$ are the eigenvectors of the three tensors, and $\left\{[*]_{i}\right\}_{i=1, \ldots, n_{\operatorname{dim}}}$ the components of the three vectors. The eigenvectors are the same for the three tensors because of the isotropy assumptions. Thus, they are fully specified by the trial state.

After some manipulations, the following nonlinear system of equations is found:

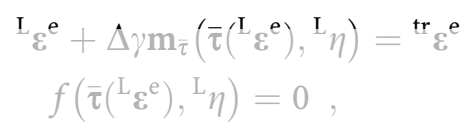

where $\Delta \gamma=\Delta t \dot{\gamma}$ is the incremental plastic multiplier, $\mathbf{m}_{\bar{\tau}}$ is the flow vector in the principal direction space (that is, $\mathbf{m}_{\tau}=\sum_{i=1}^{n_{\mathrm{dim}}}\left[\mathbf{m}_{\bar{\tau}}\right]_{j} \mathbf{n}_{\mathrm{tr}}^{i} \otimes \mathbf{n}_{\mathrm{tr}}^{i}$ ), the isotropic functions $\mathbf{m}_{\bar{\tau}}$ and $f$ are expressed as functions of $\bar{\tau}$, and $\bar{\tau}$ is given by the hyperelastic relationship

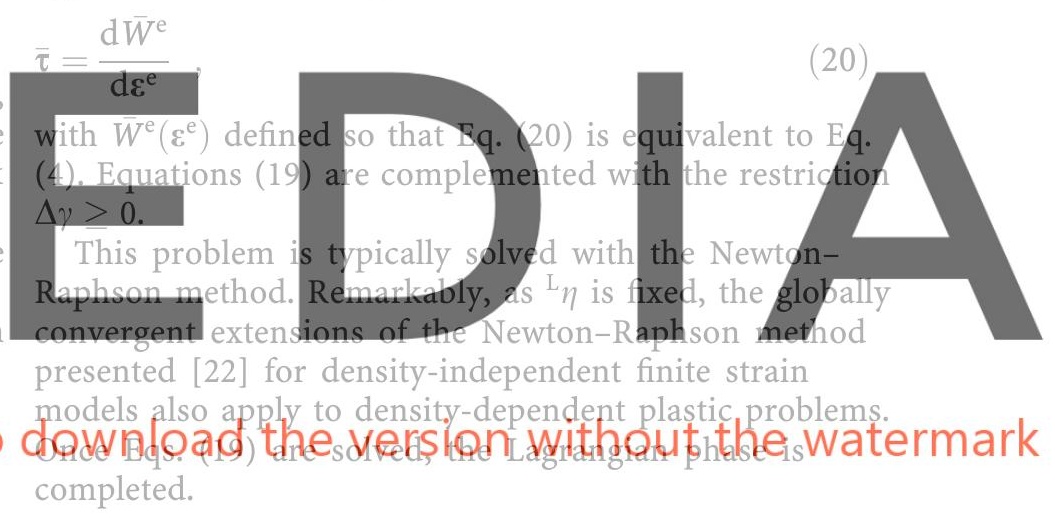

\subsection{2}

\section{Convective phase}

During the convective phase, the transport equations (14) are integrated numerically with a Godunov-like scheme $[13,24]$. The application of this scheme to quasi-static problems is compared with other alternatives in [13]. This scheme circumvents the computation of gradients with respect to the spatial coordinates present in the transport equations, the main source of trouble when discretized problems are considered. The only difference between the present application, with hyperelastic-plastic models, and that presented in $[13,24]$, with hypoelastic-plastic models, is that here it is applied to the convection of the elastic left Cauchy-Green tensor instead of the Cauchy stresses. The key points of the implementation are presented in the following.

The transport equations (14) contains seven equivalent scalar equations, one for each component of $\mathbf{b}^{\mathrm{e}}$ and one for $\eta$. Therefore, the scheme is devised for a general scalar convective equation

$\left.\frac{\partial u}{\partial t}\right|_{\chi}+\mathbf{c} \nabla_{\mathbf{x}} u=0$ 
with $u(t, \mathbf{x})$ representing the different components of $\mathbf{b}^{\mathrm{e}}$ and $\eta$. The initial condition for Eq. (21) is the Lagrangian value ${ }^{\mathrm{L}} u$.

The basic idea of the scheme is to divide every finite element in various zones, each of them corresponding to the influence domain of a Gauss point. In two-dimensional problems, as those presented in this work, each zone has an area $A$ and $n_{\text {edg }}$ edges. Then, at each Gauss point, the following explicit update equation is applied$$
{ }^{n+1} u={ }^{\mathrm{L}} u-\frac{\Delta t}{2 A} \sum_{\mathrm{i}_{\mathrm{edg}}=1}^{\mathrm{n}_{\text {edg }}} \mathrm{I}_{\mathrm{i}_{\text {edg }}}\left({ }^{\mathrm{L}} u_{\mathrm{i}_{\text {edg }}^{\mathrm{c}}}^{\mathrm{c}}-{ }^{\mathrm{L}} u\right)\left(1-\operatorname{sign}\left(\mathrm{I}_{\mathrm{i}_{\text {edg }}}\right)\right),
$$

where ${ }^{\mathrm{L}} u_{\mathrm{i}_{\text {edg }}^{\mathrm{c}}}^{\mathrm{c}}$ is the value of the variable $u$ in the contiguous Gauss point across edge $i_{\text {edg }}$ and $I_{i_{\text {edd }}}$ is the flux of convective velocity $\mathrm{c}$ across edge $i_{\text {edg. }}$. This scheme leads to a very simple algorithm. Moreover, as the space discretization and the convective velocity are the same for the different scalar transport equations, the major part of the computations are common to all of them.

Once Eq. (22) is applied to the six components of $\mathbf{b}^{\mathrm{e}}$ and $\eta$ the convective phase is completed: the values of ${ }^{n+1} \mathbf{b}^{\mathrm{e}}$ and ${ }^{n+1} \eta$ are determined. The stress values ${ }^{n+1} \tau$ and ${ }^{n+1} \sigma$ are computed with Eqs. (4) and (5).

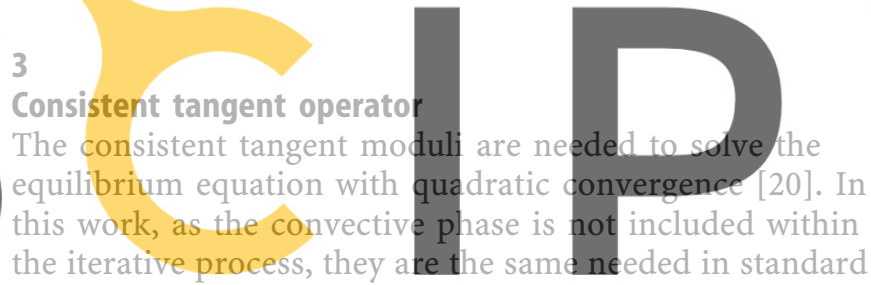
Lagrangian approaches. However, due to the density-

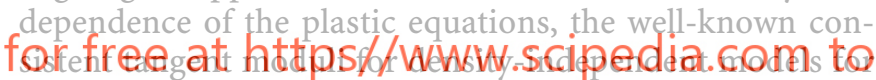
multiplicative finite strain problems [3] results incomplete. In the following, the complete expression for densitydependent plastic models is presented.

The consistent tangent moduli, $\mathbf{c}$, are the linearization of the Kirchhoff stresses obtained from the Lagrangian phase, ${ }^{\mathrm{L}} \tau$, with respect to the gradient of the incremental particle displacements, $\nabla_{n_{\mathbf{X}}}\left({ }^{n+1} \Delta \mathbf{u}\right)[3,25]$. They are found by applying the chain rule to equation $(18)_{2}$, which leads to

$\mathbf{c}=\sum_{i=1}^{n_{\mathrm{dim}}} \sum_{j=1}^{n_{\mathrm{dim}}}[\mathbf{a}]_{i j} \mathbf{n}_{\mathrm{tr}}^{i} \otimes \mathbf{n}_{\mathrm{tr}}^{i} \otimes \mathbf{n}_{\mathrm{tr}}^{j} \otimes \mathbf{n}_{\mathrm{tr}}^{j}+2 \sum_{i=1}^{n_{\mathrm{dim}}}[\mathrm{L} \bar{\tau}]_{i} \hat{\mathbf{c}}_{\mathrm{tr}}^{i}$,

with the first term corresponding to the linearization of ${ }^{\mathrm{L}} \bar{\tau}$ and the second one to the linearization of $\left\{\mathbf{n}_{\mathrm{tr}}^{i}\right\}_{i=1, \ldots, \mathrm{n}_{\mathrm{dim}}}$, and where the tensors $\left\{\hat{\mathbf{c}}_{\mathrm{tr}}^{i}\right\}_{i=1, \ldots, \mathrm{n}_{\mathrm{dim}}}$ depend on $\left\{\mathbf{n}_{\text {tr }}^{i}\right\}_{i=1, \ldots, n_{\text {dim }}}$ and ${ }^{\operatorname{tr}} \boldsymbol{\varepsilon}^{\mathrm{e}}$ [3], and $\mathbf{a}$ is a matrix of order $n_{\text {dim }}$ defined as

$\mathbf{a}=\frac{\mathrm{d}^{\mathrm{L}} \bar{\tau}}{\mathrm{d}^{\mathrm{tr}} \boldsymbol{\varepsilon}^{\mathrm{e}}}$

Equation (23) has the same expression for densitydependent and density-indepedent plastic models because the density does not affect the application of the chain rule nor the trial state. In fact, the influence of the density is restricted to the values of ${ }^{\mathrm{L}} \bar{\tau}$ and $\mathbf{a}$ in plastic steps, i.e. when the trial state is not admissible. In elastic steps, the matrix a is the Hessian of $\bar{W}^{\mathrm{e}}$,

$\mathbf{a}=\left.\frac{\mathrm{d}^{2} \bar{W}^{\mathrm{e}}}{\mathrm{d} \boldsymbol{\varepsilon}^{\mathrm{e}^{2}}}\right|_{\boldsymbol{\varepsilon}^{\mathrm{e}}=\mathrm{r}^{\mathrm{r}} \boldsymbol{\varepsilon}^{\mathrm{e}}}$

The value of ${ }^{\mathrm{L}} \bar{\tau}$ is obtained, in both elastic and plastic steps, directly from the hyperelastic relationship. Therefore, only the expression of a for plastic steps needs to be determined.

In order to do that it is useful to rephrase the dependence of ${ }^{\mathrm{L}} \eta$ on ${ }^{\mathrm{L}} \mathbf{f}$, Eq. (16), as

${ }^{\mathrm{L}} \eta={ }^{n} \hat{\eta} \exp \left(-\operatorname{tr}\left({ }^{\mathrm{tr}} \boldsymbol{\varepsilon}^{\mathrm{e}}\right)\right)$,

with ${ }^{n} \hat{\eta}={ }^{n} \eta \sqrt{\operatorname{det}\left({ }^{n} \mathbf{b}^{\mathrm{e}}\right)}$ a known value from the previous

time step and where $\operatorname{tr}(*)$ means trace of $*$ (that is,

$\left.\sum_{i=1}^{n_{d} \operatorname{dim}}[*]_{i}\right)$. Equation (26) is found by applying the deter-

minant function to both sides of Eq. (17) and substituting

Eq. (18) ${ }_{1}$ into it.

A more convenient expression of $\mathbf{a}$ is found from its definition, Eq. (24), and the application of the chain rule:

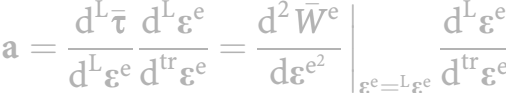

(27)

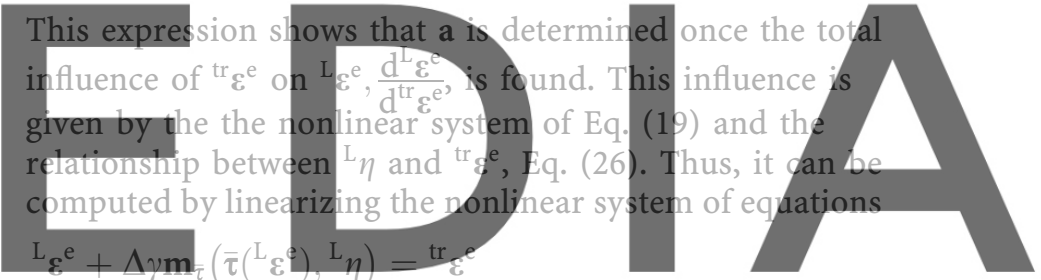

\section{download the versipenpwitholet)the watermark}

Once $d^{L} \varepsilon^{e} / d^{t r} \varepsilon^{e}$ is determined, the expression of a is found substituting it into Eq. (27). The result can be rearranged as

$\mathbf{a}=\mathbf{a}_{\eta}$-indep $+\mathbf{a}_{\eta}$

with $\mathbf{a}_{\eta \text {-indep }}$ equal to the standard consistent moduli for density-independent plastic models,

$\mathbf{a}_{\eta-\text { indep }}=\tilde{\mathbf{G}}-\frac{\tilde{\mathbf{G}} \mathbf{m}_{\bar{\tau}} \nabla_{\bar{\tau}} f^{\mathrm{T}} \tilde{\mathbf{G}}}{\nabla_{\bar{\tau}} f^{\mathrm{T}} \tilde{\mathbf{G}} \mathbf{m}_{\bar{\tau}}}$,

where

$\tilde{\mathrm{G}}=\left(\left(\frac{\mathrm{d}^{2} \bar{W}^{\mathrm{e}}}{\mathrm{d} \boldsymbol{\varepsilon}^{\mathrm{e}^{2}}}\right)^{-1}+\Delta \gamma \nabla_{\bar{\tau}} \mathbf{m}_{\bar{\tau}}\right)^{-1}$

and

$$
\begin{aligned}
\mathbf{a}_{\eta}= & \eta \Delta \gamma\left(\tilde{\mathbf{G}}-\frac{\tilde{\mathbf{G}} \mathbf{m}_{\bar{\tau}} \nabla_{\bar{\tau}} f^{\mathrm{T}} \tilde{\mathbf{G}}}{\nabla_{\bar{\tau}} f^{\mathrm{T}} \tilde{\mathbf{G}} \mathbf{m}_{\bar{\tau}}}\right) \frac{\partial \mathbf{m}_{\bar{\tau}}}{\partial \eta} \mathbf{1}_{1, n_{\mathrm{dim}}} \\
& +\eta \frac{\partial f}{\partial \eta} \frac{\tilde{G} \mathbf{m}_{\bar{\tau}}}{\nabla_{\bar{\tau}} f^{\mathrm{T}} \tilde{G} \mathbf{m}_{\bar{\tau}}} \mathbf{1}_{1, n_{\mathrm{dim}}}
\end{aligned}
$$

a term that takes into account the influence of the density.

All quantities involved in the computation of a, Eqs.

(30-32), are evaluated at the end of the Lagrangian phase. 
In the density-independent case, symmetric tangent moduli are obtained for associative material models. On the contrary, unsymmetric moduli are found with all density-dependent material models because $\mathbf{a}_{\eta}$ is, in general, unsymmetric. For this reason, in density-dependent plasticity, unsymmetric linear solvers have to be used in order to keep the characteristic quadratic convergence of the Newton-Raphson method.

On the other hand, it is important to remark that the expression of $\mathbf{a}_{\eta}$ can be computed with just a few more matrix-vector products than the standard $\mathbf{a}_{\eta \text {-indep }}$, compare Eqs. (32) and (30), and, as expected, the additional information

$\frac{\partial \mathbf{m}_{\bar{\tau}}}{\partial \eta}$ and $\frac{\partial f}{\partial \eta}$.

\section{1}

\section{Numerical differentiation}

A key point in the computation of consistent tangent moduli in density-independent plasticity is the computation of flow vector and hardening law derivatives. Reference [18] presents an analysis of different numerical differentiation schemes to compute these derivatives and references $[23,26]$ show the application to different nontrivial elastoplastic models and time-integration rukes. The main conclusion of these works is that simple first and second order difference schemes do not disturb the quadratic convergence of the $\mathrm{N}$ vided that the stepsize is fixec in that references it is shown t is an efficient alternative to-analytical derivatives even if are readily available.

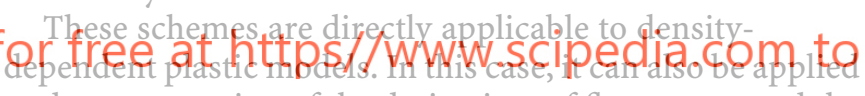
to the computation of the derivatives of flow vector and the yield function with respect to the relative density, see Eq. (33), a situation of special interest when realistic constitutive laws are considered. In this work, a first order difference scheme with a relative stepsize equal to $10^{-5}$ has been used to approximate these derivatives.

\section{4}

\section{Numerical simulations}

In this section, the proposed approach is applied to the simulation of several powder compaction problems. In the first two cases the attention is focused in the comparison of the present numerical results with numerical and experimental ones reported in the literature. The first one is solved with a pure Lagrangian approach, and the second one using the ALE formulation presented in Sect. 2. Specific analysis related with the application of the ALE formulation, the definition of the globally convergent schemes for the plastic corrector step, and the computation of the consistent tangent moduli via numerical differentiation schemes can be found in references [14, 18, 22, 23].

The powder material is modelled with the Hencky's hyperelastic law, which leads to a linear relationship between $\bar{\tau}$ and $\boldsymbol{\varepsilon}^{\mathrm{e}}$, and associate plasticity with the following elliptic yield function $[1,27]$ $f_{\text {ellip }}(\tau, \eta)=2 J_{2}(\tau)+a_{1}(\eta)\left(\frac{I_{1}(\tau)}{3}\right)^{2}-\frac{2}{3} a_{2}(\eta)\left(\sigma_{y}\right)^{2}$,

with $I_{1}(\tau)$ equal to the first invariant of $\tau, J_{2}(\tau)$ equal to the second invariant of the deviatoric part of $\tau$, and the density-dependent parameters

$a_{1}(\eta)=\left\{\begin{array}{ll}\left(\frac{1-\eta^{2}}{2+\eta^{2}}\right)^{n_{1}} & \eta<1 \\ 0 & \eta \geq 1\end{array}\right.$ and
$a_{2}(\eta)= \begin{cases}\left(\frac{0.02 \eta_{0}}{1-0.98 \eta_{0}}\right)^{n_{2}} & \eta \leq \eta_{0} \\ \left(\frac{\eta-0.98 \eta_{0}}{1-0.98 \eta_{0}}\right)^{n_{2}} & \eta>\eta_{0}\end{cases}$

The dependence of $a_{1}$ and $a_{2}$ on $\eta$ for the material parameters presented in Table 2 is depicted in Fig. 2. The trace of the yield function on the meridian plane $p_{\tau}-q_{\tau}$, with $p_{\tau}=-\frac{I_{1}(\tau)}{3}$ and $q_{\tau}=\sqrt{3 J_{2}(\tau)}$, for different relative densities are depicted in Fig. 3. Note that $f_{\text {ellip }}$ becomes the von Mises yield function for $\eta \geq 1$.

4.1

Compaction of a plain bush component

The first example is the uniaxial compaction of a plain

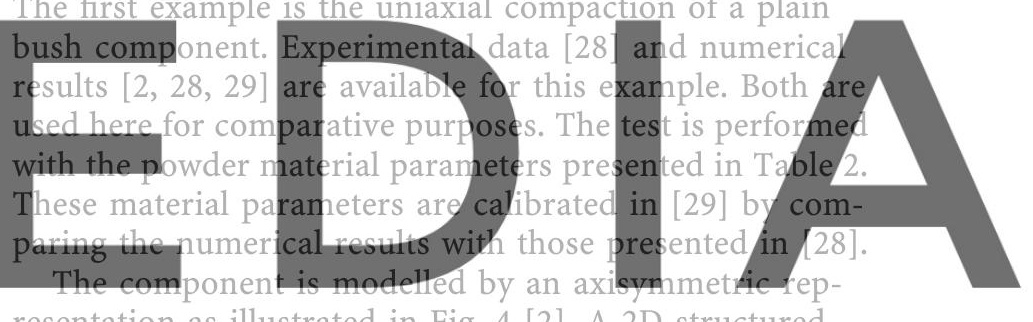
resentation as illustrated in Fig. 4 [2]. A 2D structured

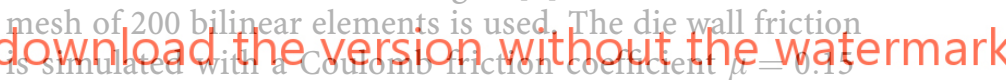

Table 2. Material parameters

\begin{tabular}{ll}
\hline$E$ & $50000 .[\mathrm{MPa}]$ \\
$v$ & 0.37 \\
$\sigma_{y}$ & $12 .[\mathrm{MPa}]$ \\
$\eta_{0}$ & 0.41 \\
$\eta_{1}$ & 0.5 \\
$\eta_{2}$ & 2.2 \\
\hline
\end{tabular}
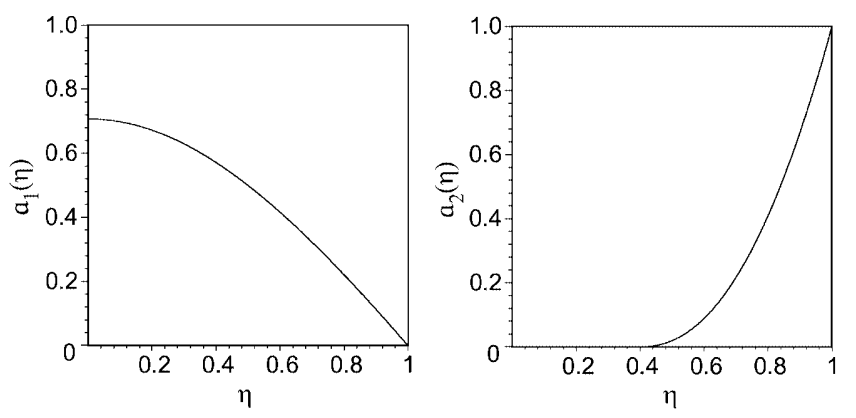

Fig. 2. Dependence of parameters $a_{1}(\eta)$ and $a_{2}(\eta)$ on the relative density, $\eta$ 
$[28,29]$ acting in the inner and outer walls of the sample, segments $\overline{\mathrm{BC}}$ and $\overline{\mathrm{DA}}$ of Fig. 4. In [29] the relative radial movement of top and bottom surfaces with respect to the punches, segments $\overline{\mathrm{AB}}$ and $\overline{\mathrm{CD}}$, is allowed, with a Coulomb friction coefficient equal to that of the lateral surfaces.

Here, following [2], the radial displacement of the top and the bottom of the sample is restrained. The vertical displacement of segment $\overline{\mathrm{AB}}$ is also set equal to zero, and a vertical displacement of $11.5 \mathrm{~mm}$ is imposed to segment $\overline{\mathrm{CD}}$, simulating the top punch movement.

The relative density profile at radius equal to $10.5 \mathrm{~mm}$ for a top punch displacement of $10 \mathrm{~mm}$ is shown in Fig. 5 . The numerical results of $[28,29]$ and the experimental data of [28] are included in the same figure. The results of the present numerical simulation are in agreement with all of them. However, as expected because of the similarities of the material model, the best agreement is found with the results of [29]. The lower relative density at the top part of the sample found by [29], with respect to the present one, and the higher values in the lower part are directly related with the different treatment of the friction effects of top and bottom punches.

\section{D}

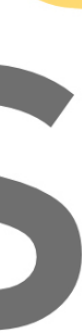

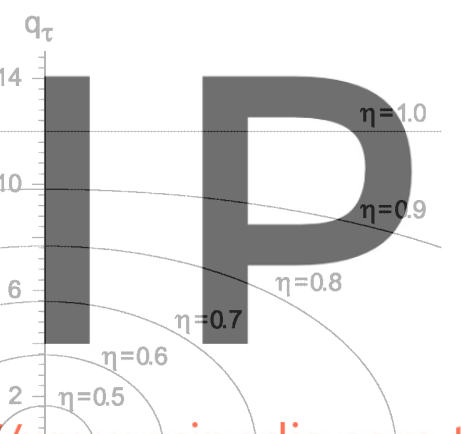

for free at https//NWWW.scipedia.com to

Fig. 3. Trace of the elliptic yield function on the meridian plane $q_{\tau}-p_{\tau}$ for different relative densities, $\eta$

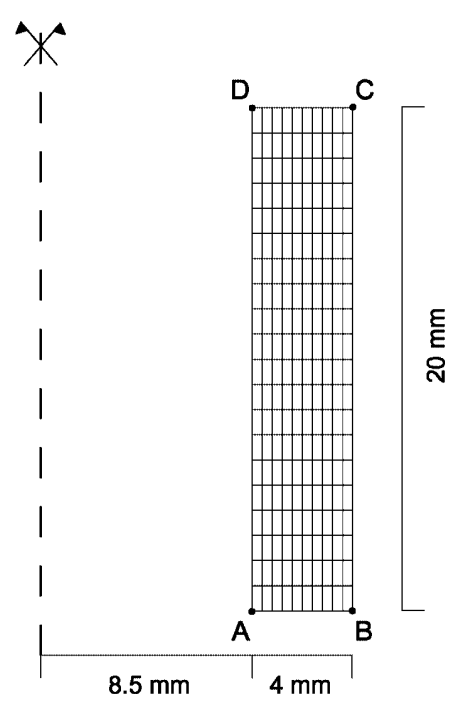

Fig. 4. Plain bush component. Problem definition (after Lewis and Khoei 1998) and computational mesh
The evolution of the vertical reaction of the punch with respect to its vertical displacement is depicted in Fig. 6. The numerical results of [2] and the experimental data of [28] are also included. In reference [2] the results obtained with different formulations are shown. Those depicted in Fig. 6 are the most similar to the experimental data. The agreement between the results of the present simulation and the experimental ones is good.

Finally, the evolution of the relative density distribution over the sample is depicted in Fig. 7. Results for different vertical top punch displacements (from 4 to $11 \mathrm{~mm}$ ) are shown. Two different relative density scales are used (one in each row) in order to show better the non-homogeneous distribution of the density. The shape of the relative density distributions are in general agreement with those

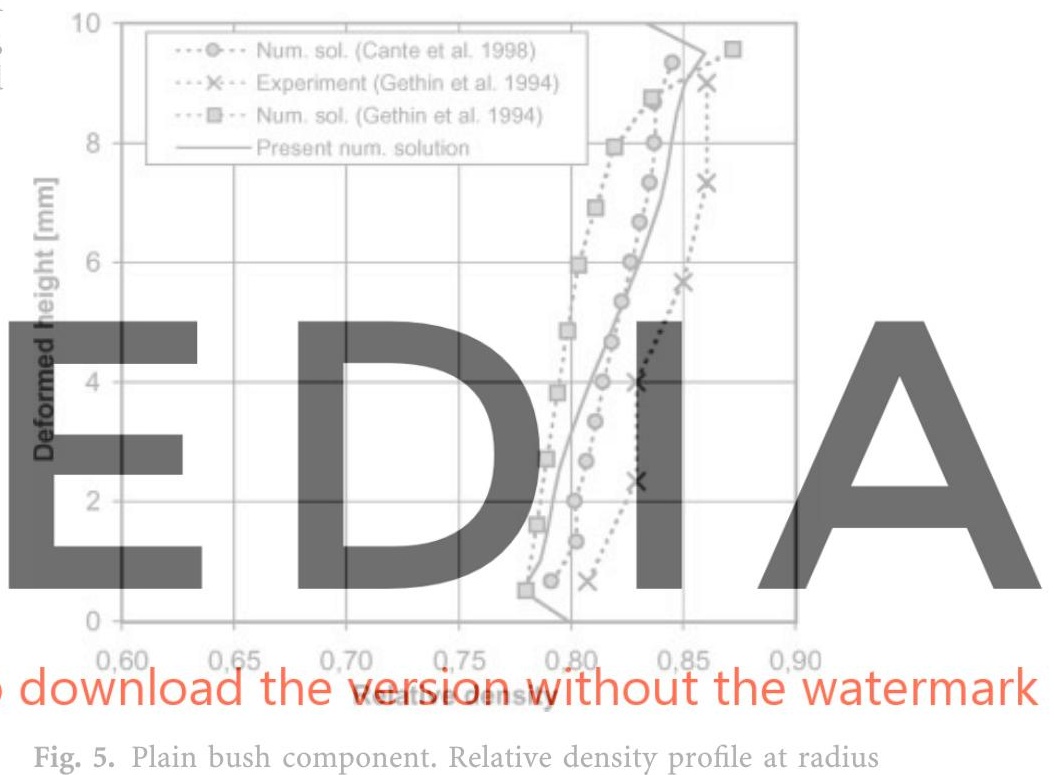

Fig. 5. Plain bush component. Relative density profile at radius equal to $10.5 \mathrm{~mm}$

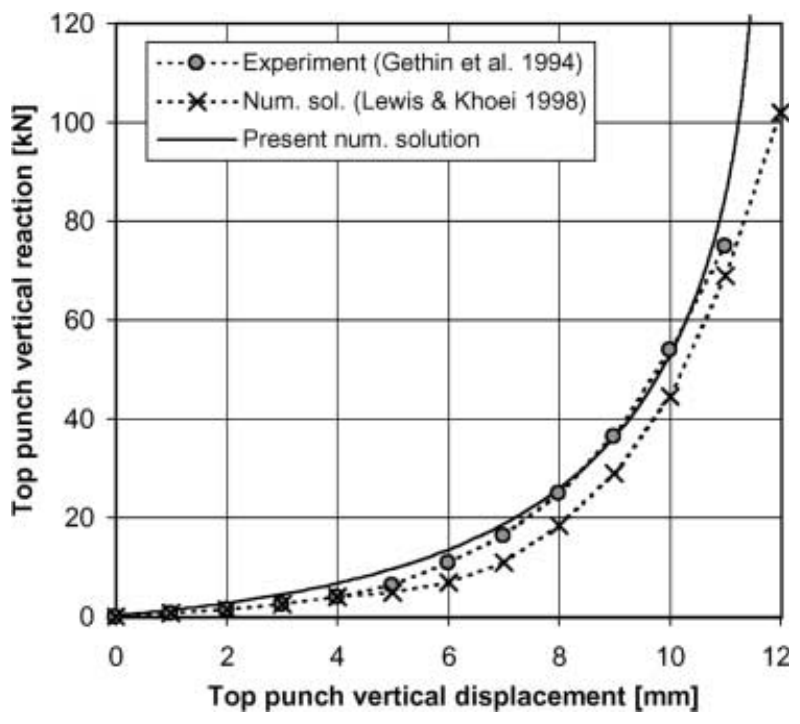

Fig. 6. Plain bush component. Relationship between top punch vertical reaction and its vertical displacement 
three tests. The die wall friction is simulated with a Coulomb friction coefficient $\mu=0.08$ acting in the segments $\overline{\mathrm{BC}}, \overline{\mathrm{CD}}, \overline{\mathrm{DE}}$ and $\overline{\mathrm{FA}}$, see Fig. 8 , and the radial displacement at the punches is restrained [2].

The analysis is performed with the powder material of Table 2, calibrated in [1] for the compaction of the plain bush component. The relationships between dimensionless vertical loads and punch displacements obtained in this work are compared with those of [2] in Fig. 9. The agreement between the two sets of curves is evident. However, the reference load is different: $392 \mathrm{kN}$ for the present results and $1550 \mathrm{kN}$ for the results of [2] (in both cases the final reaction of the top punch in the doublepunch compaction test). This difference can be related with the different modelling approach (dynamic versus static) and, especially, with the great difference between

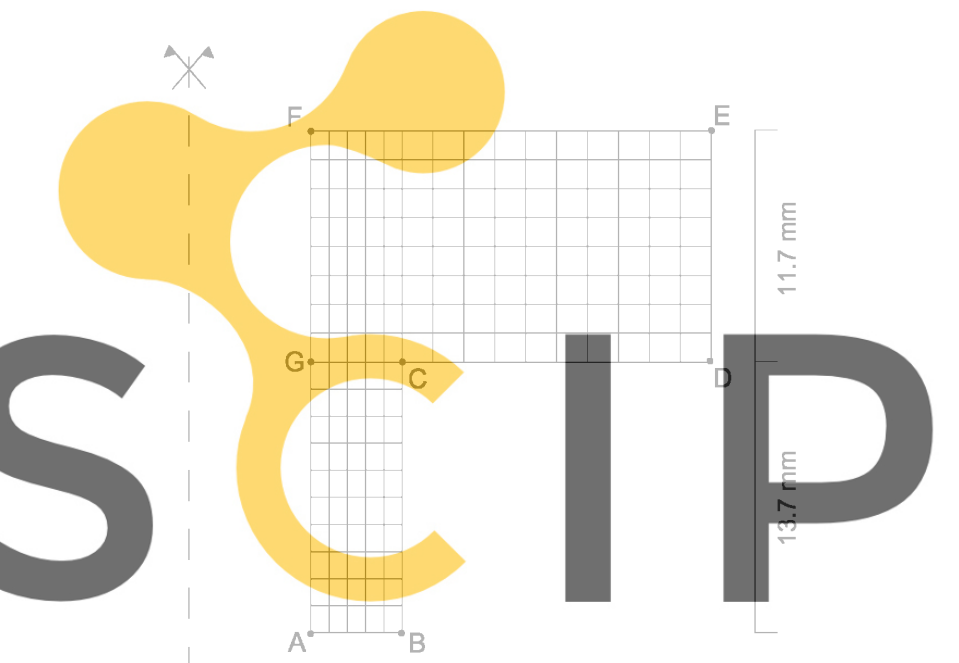

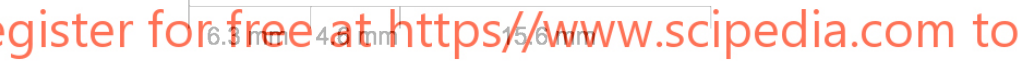

Fig. 8. Flanged component. Problem definition (after Lewis and Khoei 1998) and computational mesh

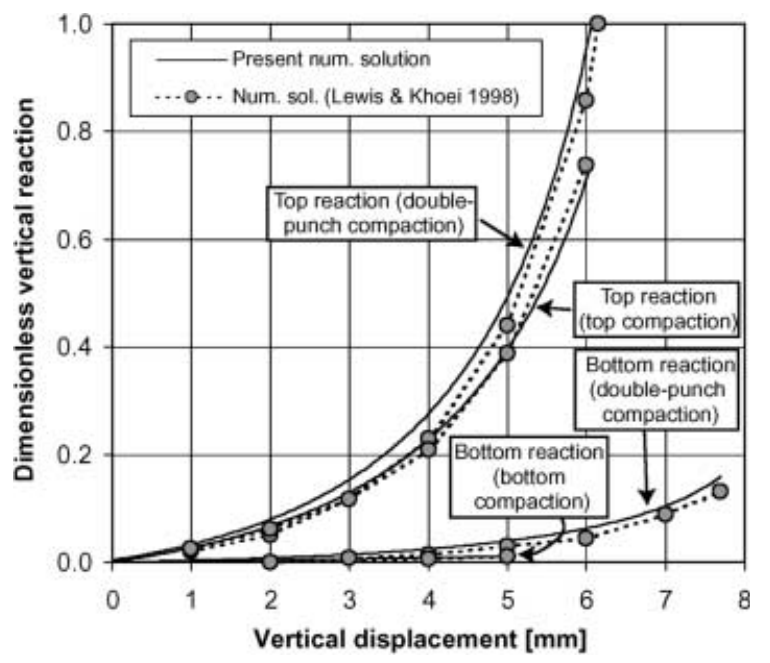

Fig. 9. Flanged component. Relationships between the vertical reactions of the punches and their vertical movements: top reaction for top punch compaction, bottom reaction for bottom punch compaction, and top and bottom reactions for doublepunch compaction the elastic moduli used in both cases (40 MPa in [2], three orders of magnitude lower than the one used here). In the following, a detailed analysis of the relative density distribution obtained in each of the three tests is presented.

In the first test, top punch compaction, the mesh region ABCG is Eulerian, and equal height elements are prescribed in the mesh region GDEF. The variation of the mass of the sample during the simulation is depicted in Fig. 10. A final loss of $0.75 \%$ is found. This small variation corresponds to the truncation error in the discretization of the convective term of the ALE formulation (both temporal and spatial discretization).

The evolution of the relative density distribution is summarized in Fig. 11(a-d). The compaction process leads to a clearly non-homogenous density distribution. As expected, higher values are found in the outer region of the sample and lower ones close to the bottom surface. A smooth transition from higher to lower densities is found. A dense zone is detected in the corner region, just over the point C, during all the process. Recall that there is not mesh distortion because, although the flux of mass is important, the mesh does not follow the material particles in the ALE formulation. The final relative density profile at $1.88 \mathrm{~mm}$ from line $\overline{\mathrm{GD}}$ is depicted in Fig. 11(e). The present results are in general agreement with those

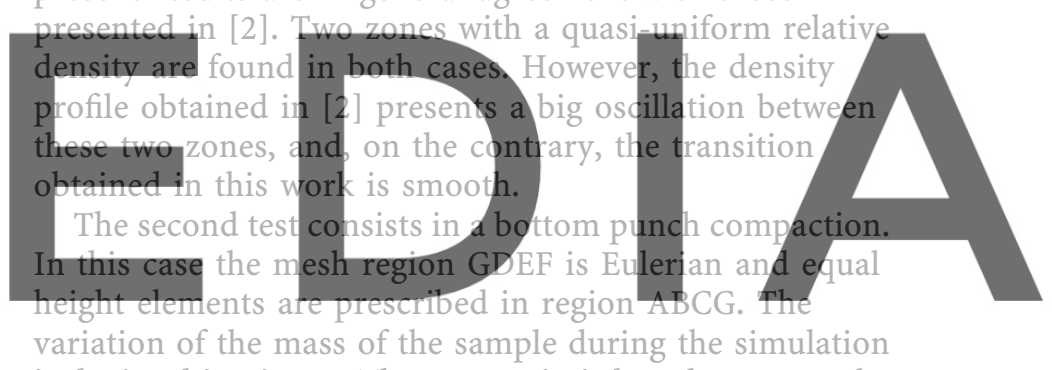

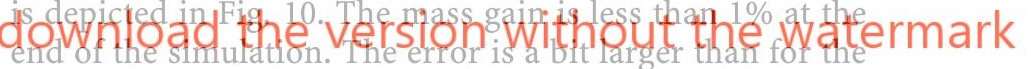

top punch test. This indicates that the convective effects are more important in this case.

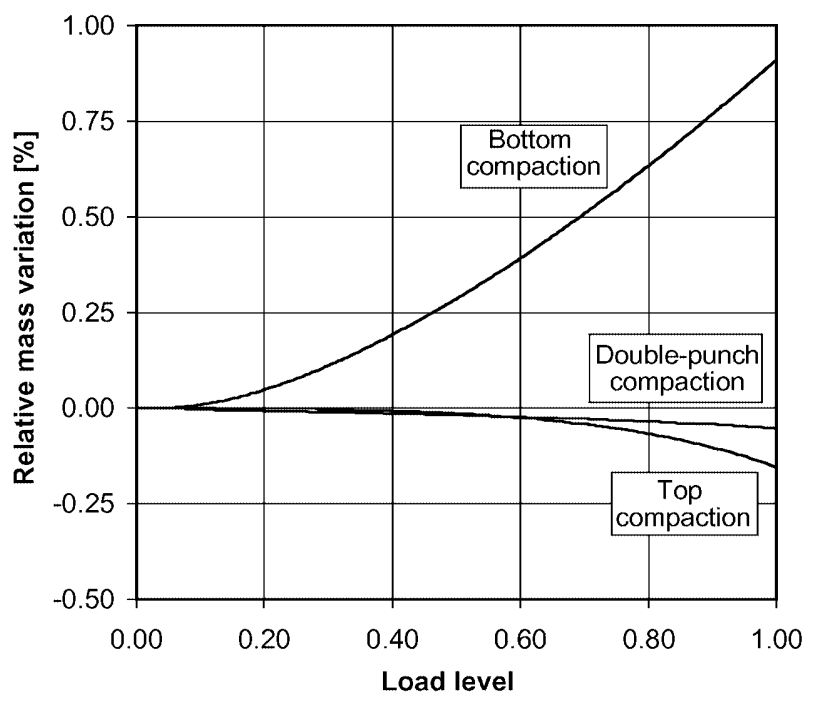

Fig. 10. Relative mass variation during the three compaction processes of the flanged component. The load levels are referred to the punch displacements imposed at the end of each test 
(a)

$\mathrm{d}_{\mathrm{b}}=1.275 \mathrm{~mm}$

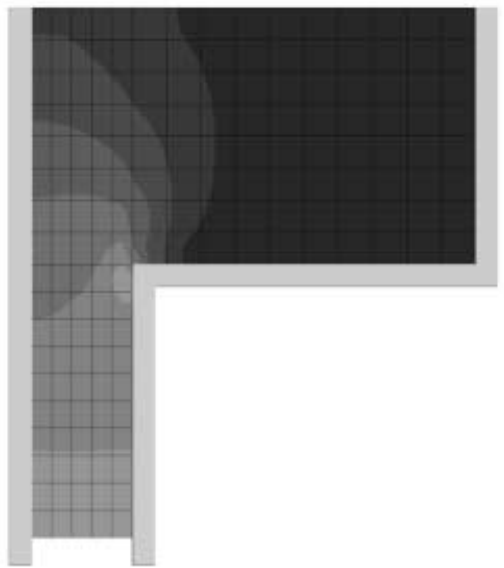

(c)

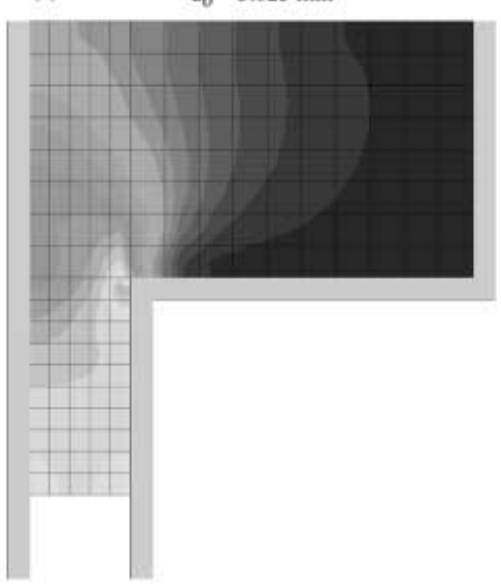

(e)

Section 1-1"

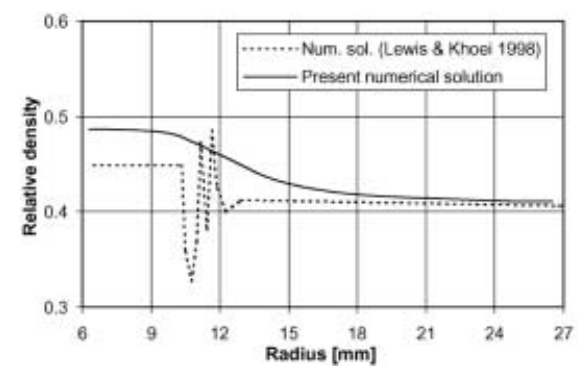

density scale are depicted is Fig. 13(a). Specific figures for each load level are also included, Fig. 13(b-d). The compaction process leads to a quasi-homogeneous density distribution during all the test, with differences less than $10 \%$. Higher values are found in the bottom of the sample and lower values close to point D. Four relative density profiles are depicted in Fig. 14. The numerical results presented in [2] and the experimental data available [30] are included in the same figure. All these results are in general agreement.

\section{3}

\section{A multi-level component}

The last example demonstrates the performance of the ALE formulation for the complicated die geometry of a multi-level component shown in Fig. 15. This geometry is used in [6] to show the applicability of an $h$-adaptive remeshing technique. It is included here to show that the
Fig. 12. Bottom punch compaction. (a-d) Relative density distribution for different bottom punch movements and (e) relative density profile at $3.9 \mathrm{~mm}$ from line $\overline{\mathrm{GD}}$ (section 1-1')

ALE formulation also work with unstructured meshes. The material parameters of the two previous examples, powder-C, are used (see Table 2).

An unstructured mesh of 410 eight-noded elements with reduced integration (four Gauss points per element) is used. The die wall friction is simulated with a Coulomb friction coefficient $\mu=0.08$ acting in the segment $\overline{\mathrm{FI}}$. The radial displacement of top and bottom punches is set equal to zero. The symmetry conditions at the segment $\overline{\mathrm{LA}}$ imply a radial displacement equal to zero and free vertical movement.

Three different compaction tests are simulated: 1) a vertical movement of $16 \mathrm{~mm}$ of the top punch (segments $\overline{\mathrm{IJ}}, \overline{\mathrm{JK}}$ and $\overline{\mathrm{KL}}$ of Fig. 15); 2) a vertical movement of $16 \mathrm{~mm}$ of the bottom punch (segments $\overline{\mathrm{AB}}, \overline{\mathrm{BC}}, \overline{\mathrm{CD}}, \overline{\mathrm{DE}}$ and $\overline{\mathrm{EF}}$ ); and 3) a simultaneous, and opposite, movement of $8 \mathrm{~mm}$ of both punches. The three compaction tests lead to the same final geometry. 

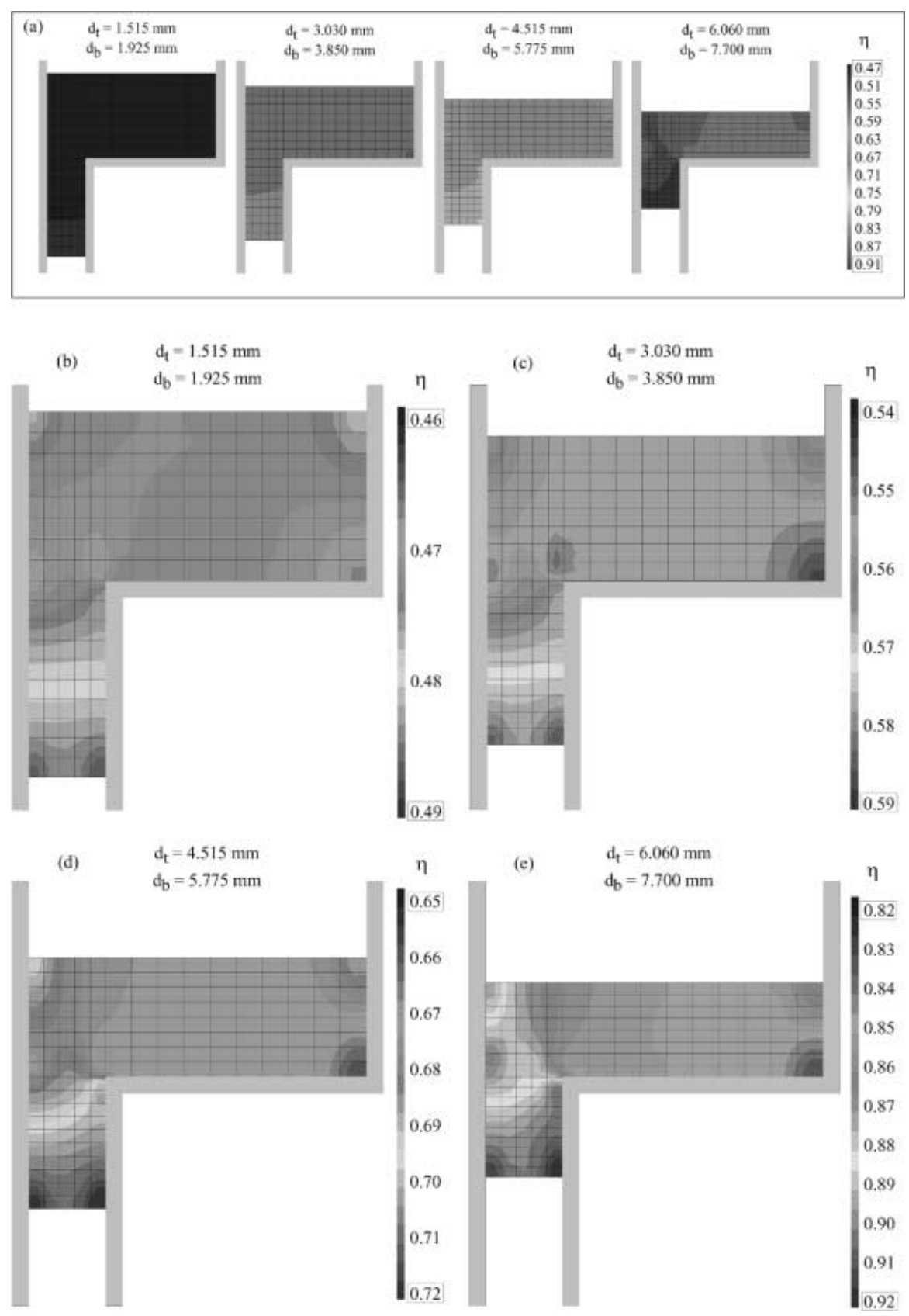

Fig. 13. Double-punch compaction. Relative density distribution for different movements of the top and bottom punches. (a) common density scale and (b-e) different scales
The movement of the mesh is prescribed in the same way in the three tests. In the mesh regions $A B C M$ and DEFG the mesh nodes are moved vertically the same as the bottom punch, and in the region HIJK the same as the top punch. In the central part of the sample, region MGHL, the vertical movement of the nodes is linearly interpolated between top and bottom movements. The radial movement of all nodes is restrained. Low relative errors of the sample mass are found in the three tests. The maximum mass reductions have been found at the end of the tests. They are $0.20 \%$ for top compaction, $0.19 \%$ for bottom compaction and $0.17 \%$ for double-punch compaction.

The final relative density distributions of the three cases are depicted in Fig. 16. As expected, the results are similar and the influence of the compaction process is basically restricted to the top and bottom parts close to the die wall.
The final relative density distributions are similar to those presented in [6]. However, as in the previous examples, the present results do not present spurious oscillations.

Moreover, the quantitative values of the relative density, which are lower than those presented in [6], are validated by the verification of the mass conservation principle.

The relative density profiles at 2 millimeters of the die wall (line $\overline{\text { FI}}$ ) are depicted in Fig. 17. In the three tests, the influence of the shape of the punches is clear. The difference between the shape of top and bottom punches is also reflected in these curves: in both tests involving top punch compaction the upper part has a quasi-uniform distribution, in contrast with the lower part, where large density differences are found even in the bottom punch compaction test. This different behaviour is directly related with the different height of the indentations of both punches. 
(a) Final configuration

(b)

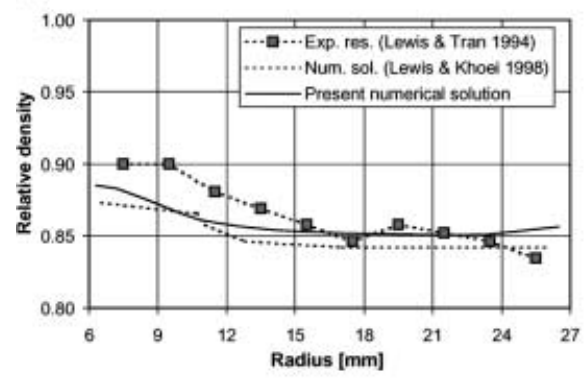

(d)

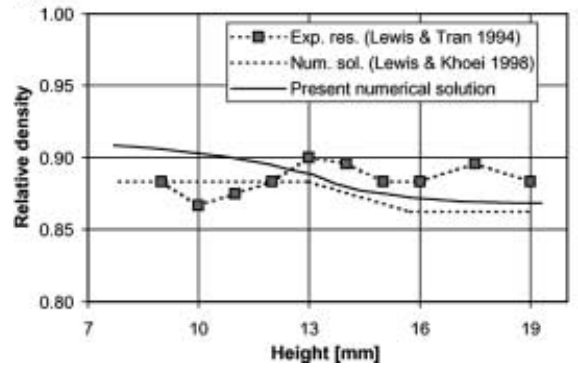

(c)

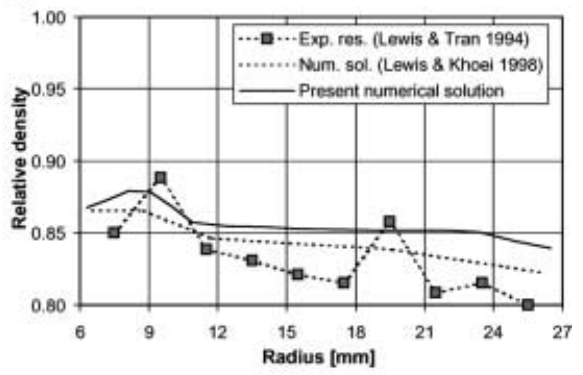

(e)

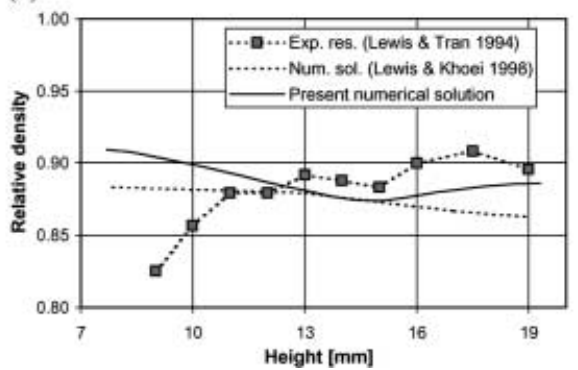

Fig. 14. Double-punch compaction. Relative density profiles at $3.47 \mathrm{~mm}$ (section 1-1') and $1.3 \mathrm{~mm}$ (section 2-2') from the line $\overline{\mathrm{GD}}$, and at radii equal to $9.37 \mathrm{~mm}$ (section 3-3') and $8.77 \mathrm{~mm}$ (section 4-4')

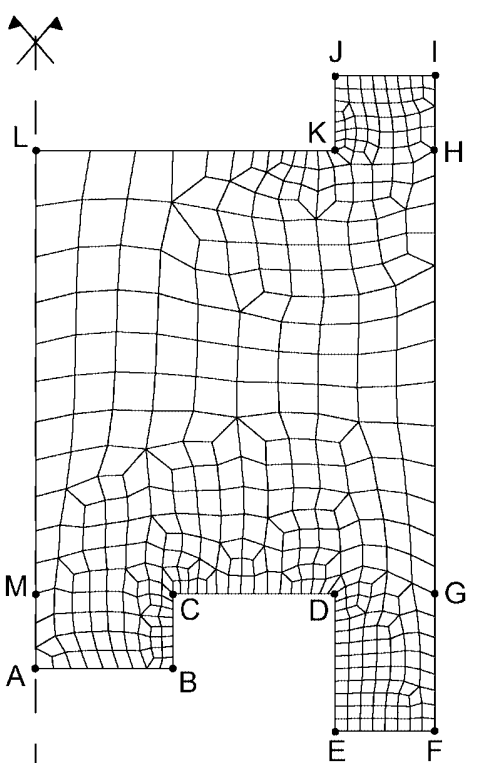

Fig. 15. Multi-level component. Problem definition (after Khoei and Lewis 1999) and computational mesh

$$
\begin{aligned}
& \overline{\mathrm{AB}}=11 \mathrm{~mm} \\
& \overline{\mathrm{CD}}=13 \mathrm{~mm} \\
& \overline{\mathrm{EF}}=8 \mathrm{~mm} \\
& \overline{\mathrm{IJ}}=8 \mathrm{~mm} \\
& \overline{\mathrm{KL}}=24 \mathrm{~mm}
\end{aligned}
$$

Vertical dimensions

$\overline{\mathrm{MA}}=\overline{\mathrm{BC}}=6 \mathrm{~mm}$

$\overline{\mathrm{DE}}=\overline{\mathrm{FG}}=11 \mathrm{~mm}$

$\overline{\mathrm{GH}}=\overline{\mathrm{LM}}=35.5 \mathrm{~mm}$

$\overline{\mathrm{HI}}=\overline{\mathrm{JK}}=6 \mathrm{~mm}$
On the other hand, note that the double-punch compaction curve gathers the main characteristics of top and bottom compaction curves, in its upper and lower halves respectively.

Horizontal dimensions

\section{Conclusions}

In this paper, a new approach for the numerical simulation of powder compaction problems is presented. The powder material is modelled with a isotropic finite strain densitydependent plastic model [5] and the powder-die friction with the Coulomb dry friction model. The constitutive equations are formulated within an ALE framework [14], which can be used to avoid the excessive mesh distortion characteristic of this type of problems. A detailed description of the numerical time-integration scheme for the constitutive equations and the corresponding expression of the consistent tangent moduli are included. Attention is focused in the simulation of three representative powder compaction problems.

The elastoplastic model consists in the hyperelastic Hencky's law and an elliptic yield function expressed in terms of the relative density and the Kirchhoff stresses [1]. In contrast with previous applications of this plastic 

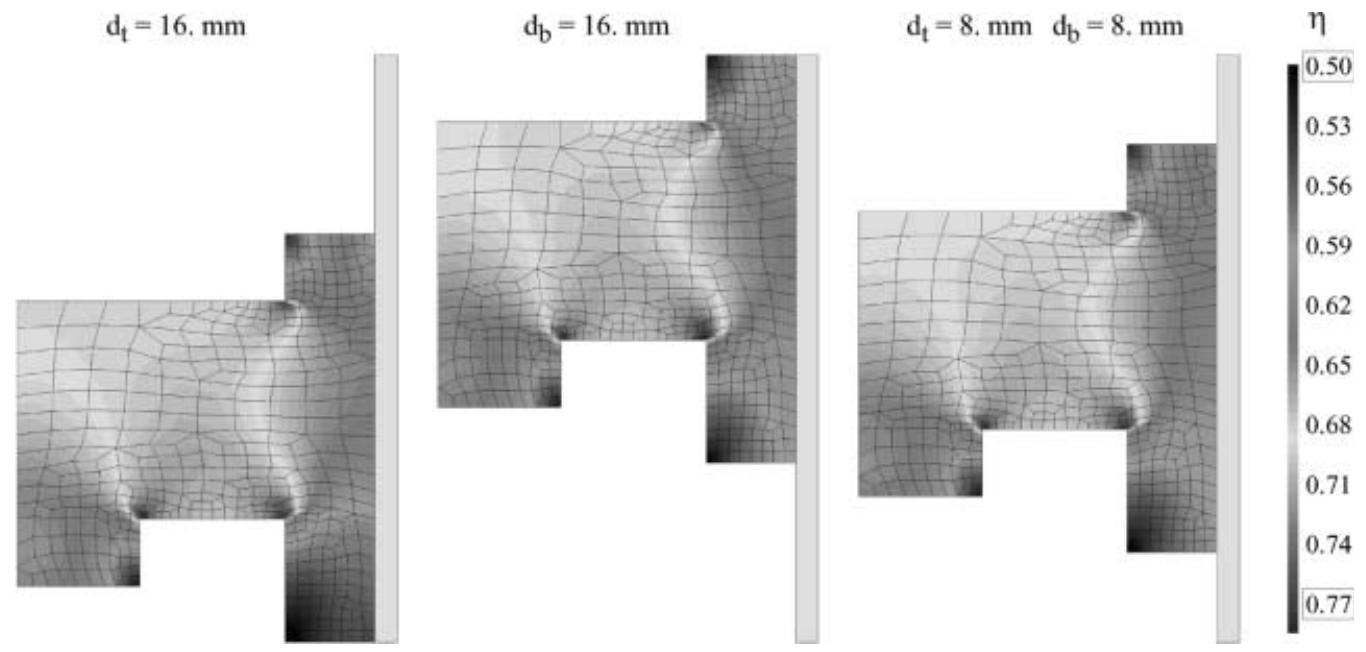

Fig. 16. Multi-level component. Relative density distribution for three different compaction processes

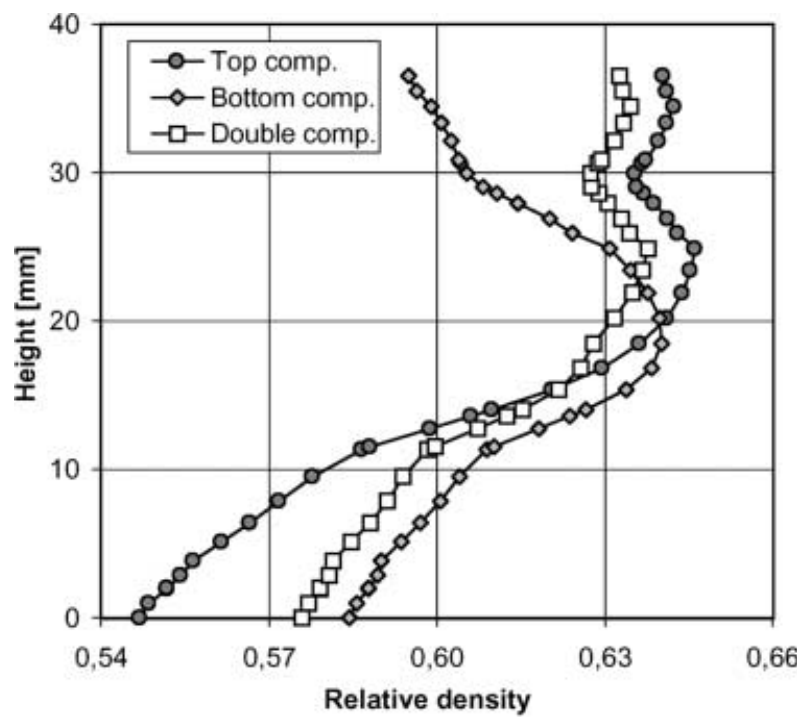

Fig. 17. Multi-level component. Relative density profile at radius equal to $30 \mathrm{~mm}$ for the three different compaction processes

model, large elastic and plastic strains are considered here. The model has been assessed with the Lagrangian simulation of the compaction of a plain bush component. The results obtained agree with the numerical and experimental data available.

The ALE formulation has been applied to several examples involving sharp boundaries and large mass fluxes between different parts of the sample. The numerical time-integration is performed in two phases: Lagrangian and convective. The Lagrangian phase is solved with the standard return mapping algorithm [3] and the convective one with a Godunov-type scheme [13]. A relative movement of the mesh with respect to the material particles is applied between the two phases with the goal of ensuring the good quality of the mesh during all the problem. Only the Lagrangian phase is computed during the iterative process for equilibrium. Remarkably, the dependence of the plastic model on the density does not modify the standard return mapping algorithm based on the exponential mapping. Moreover, it is shown that the computation of the consistent tangent moduli involves only few additional computations with respect to the well known expression for density-independent models. The remeshing and the convective phase are computed once per load increment, with the converged results. The use of simple remeshing techniques (as those used here) and the explicit character of the Godunov-type scheme guarantee a low computational overhead of the proposed ALE approach with respect to the standard Lagrangian formulation.

On the other hand, the efficiency of the proposed approach is complemented with the high accuracy of its results. It has been shown that the mass conservation principle is verified exactly in Lagrangian computations because of the complete kinematic description of the deformation process and the material version of the conservation principle (which does not need an approximation for time-integration). In the ALE examples, the mass conservation principle has been verified with very low relative errors (less than $1 \%$ for all compaction processes). This represents a significant improvement with respect to previous results based on non-adaptive and $h$-adaptive Lagrangian approaches found in the literature. Because of the high computational performance of the present approach, it has been possible to perform some detailed analysis. The present results agree with the experimental data available and the relative density distributions do not present spurious oscillations, even in compaction tests involving high convective effects.

\section{References}

1. Oliver J, Oller S, Cante JC (1996) A plasticity model for simulation of industrial powder compaction processes. Int. J. Solids Structures 33(20-22): 3161-3178

2. Lewis RW, Khoei AR (1998) Numerical modelling of large deformation in metal powder forming. Comp. Meth. Appl. Mech. Eng. 159: 291-328 
3. Simo JC (1992) Algorithms for static and dynamic multiplicative plasticity that preserve the classical return mapping schemes of the infinitesimal theory. Comp. Meth. Appl. Mech. Eng. 99: 61-112

4. Simo JC (1998) Numerical analysis and simulation of plasticity. In: Handbook of Numerical Analysis, Vol. VI, Ciarlet PG, Lions JL (eds). Elsevier, Amsterdam, pp. 179-499

5. Pérez-Foguet A, Rodríguez-Ferran A, Huerta A (2001) Consistent tangent matrices for density-dependent finite plasticity models. Int. J. Numer. Anal. Meth. Geomech. 25: 1045-1075

6. Khoei AR, Lewis RW (1999) Adaptive finite element remeshing in a large deformation analysis of metal powder forming. Int. J. Numer. Meth. Eng. 45: 801-820

7. Cante JC, Oliver J, Hernández R (1999) Simulación numérica de las etapas de transferencia y prensado del proceso de compactación de pulvimateriales. In: IV Congreso de Métodos Numéricos en Ingeniería, Abascal R, Domínguez J, Bugeda G (eds), SEMNI, Spain

8. Donéa J, Fasoli-Stella P, Giuliani S (1977) Lagrangian and Eulerian finite element techniques for transient fluid structure interaction problems. In: Transactions of the 4th Int. Conference on Structural Mechanics in Reactor Technology, paper B1/2, San Francisco

9. Huerta A, Liu WK (1988) Viscous flow with large free surface motion. Comp. Meth. Appl. Mech. Eng. 69: 277-324

10. Liu WK, Belytschko T, Chang H (1986) An arbitrary Lagrangian-Eulerian finite element method for path-dependent materials. Comp. Meth. Appl. Mech. Eng. 58: 227-245

11. Benson DJ (1986) An efficient, accurate, simple ALE method for nonlinear finite element programs. Comp. Meth. Appl. Mech. Eng. 72: 305-350

12. Ghosh S, Kikuchi N (1991) An arbitrary Lagrangian-Eulerian finite element method for large deformation analysis of elastic-viscoplastic solids. Comp. Meth. Appl. Mech. Eng. 86: 127-188

13. Rodríguez-Ferran A, Casadei F, Huerta A (1998) ALE stress update for transient and quasistatic processes. Int. J. Numer. Meth. Eng. 43: 241-262

14. Rodríguez-Ferran A, Pérez-Foguet A, Huerta A (2002) Arbitrary Lagrangian-Eulerian (ALE) formulation for hyperelastoplasticity. Int. J. Numer. Meth. Eng. 53: 1831-1851

15. Gu C, Kim M, Anand L (2001) Constitutive equations for metal powders: application to powder forming processes. Int. J. Plasticity 17: 147-209

16. Meschke G, Liu WN (1999) A re-formulation of the exponential algorithm for finite strain plasticity in terms of Cauchy stresses. Comp. Meth. Appl. Mech. Eng. 173: 167-187

17. Alfano G, Rosati L, Valoroso N (2001) A tangent-secant approach to rate-independent elastoplasticity: formulations and computational issues. Comp. Meth. Appl. Mech. Eng. 179: 379-405

18. Pérez-Foguet A, Rodríguez-Ferran A, Huerta A (2000) Numerical differentiation for local and global tangent operators in computational plasticity. Comp. Meth. Appl. Mech. Eng. 189: 277-296

19. Luccioni LX, Pestana JM, Taylor RL (2001) Finite element implementation of non-linear elastoplastic constitutive laws using local and global explicit algorithms in automatic error control. Int. J. Numer. Meth. Eng. 50: 1191-1212

20. Simo JC, Taylor RL (1985) Consistent tangent operators for rate-independent elastoplasticity. Comp. Meth. Appl. Mech. Eng. 48: 101-118

21. Armero F, Pérez-Foguet A (2002) On the formulation of closestpoint projection algorithms in elastoplasticity. Part I: the variational structure. Int. J. Numer. Meth. Eng. 53: 297-329

22. Pérez-Foguet A, Armero F (2002) On the formulation of closest-point projection algorithms in elastoplasticity. Part II: globally convergent schemes. Int. J. Numer. Meth. Eng. 53: 331-374

23. Pérez-Foguet A, Rodríguez-Ferran A, Huerta A (2000) Numerical differentiation for non-trivial consistent tangent matrices: an application to the MRS-Lade model. Int. J. Numer. Meth. Eng. 48: 159-184

24. Huerta A, Casadei F, Donea J (1995) ALE stress update in transient plasticity problems. In: Proceedings of the Fourth International Conference on Computational Plasticity (COMPLAS IV), Vol. 2, pp. 1865-1876

25. Simo JC, Hughes TJR (1998) Computational Inelasticity, Springer-Verlag.

26. Pérez-Foguet A, Rodríguez-Ferran A, Huerta A (2001) Consistent tangent matrices for substepping schemes. Comp. Meth. Appl. Mech. Eng. 190(35-36): 4627-4647

27. Oliver J, Oller S, Cante JC (1992) Numerical simulation of uniaxial compaction processes in powder materials. In: Proceedings of the International Congress on Numerical Methods in Engineering and Applied Sciences, Chile, pp 1227-1286

28. Gethin DT, Lewis RW (1994) Finite element modelling of powder compaction and its experimental validation. In: Powder Metallurgy 94, World Congress, Paris, pp. 689-692

29. Cante JC, Oliver J, Oller S (1998) Simulación numérica de procesos de compactación de pulvimateriales. Parte 2: Validación y Aplicaciones Industriales. Revista Internacional de Métodos Numéricos para Cálculo y Diseño en Ingeniería 14(1): 101-116

30. Lewis RW, Tran DV (1994) Finite element approach to problems in particulate media with special reference to powder metal forming. Bull. Tech. Univ. Istambul 47: 295-310 